\title{
Efficacy of Narrow Band Imaging System Combined With Magnifying Endoscopy for Differentiating Type Ila Early Gastric Cancer From Adenoma
}

\author{
Hyung Hun Kimª, Moo In Park, , Jeong Moon Choi ${ }^{\mathrm{a}}$, Seun Ja Park, Won Moon ${ }^{\mathrm{a}}$
}

\begin{abstract}
Background: It is not always possible for endoscopists to differentiate early gastric cancer from adenoma in 0-IIa type neoplasia. The aim of this study was to assess the relationships between images obtained with a narrow band image system combined with magnifying endoscopy (MENBI) and histological findings, especially vascular patterns, to distinguish adenoma from type IIa early gastric cancer (EGC IIa).
\end{abstract}

Methods: We postoperatively confirmed and evaluated 46 elevated lesions, 32 adenomas and 14 EGC IIa in patients who had undergone endoscopic submucosal dissection. We randomly selected three sites from each neoplasm. The selected sites were classified as four irregular microvascular patterns (IMVPs). In addition, the selected sites were divided into two groups based on the presence of corkscrews.

Results: Regarding IMVP subcategories, (1) slight intrastructural irregular microvascular patterns (ISIMVPs) accounted for $84 \%$, (2) severe ISIMVPs accounted for 6\%, (3) fine networks (FNs) accounted for $10 \%$, and (4) corkscrews accounted for 0 of cases in the adenomas. The corresponding proportions in the EGC IIa were (1) $24 \%$, (2) $31 \%$, (3) 45\%, and (4) 0. Slight ISIMVPs, severe ISIMVPs, and FNs reliably distinguished the two diseases: $\mathrm{P}<$ 0.001 for slight ISMVPs; $\mathrm{P}<0.001$ for severe ISIMVPs; $\mathrm{P}<0.001$ for FNs. The presence of corkscrews was observed in 9.5\% of EGC IIa and 0 of adenoma cases $(\mathrm{P}=0.008)$.

Conclusions: MENBI can be used to differentiate EGC IIa from

Manuscript accepted for publication September 2, 2011

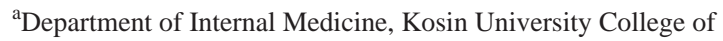
Medicine, Busan, Korea

${ }^{\mathrm{b} C o r r e s p o n d i n g ~ A u t h o r: ~ M o o ~ I n ~ P a r k, ~ D e p a r t m e n t ~ o f ~ I n t e r n a l ~ M e d i c i n e, ~}$ Kosin University College of Medicine, 34 Amnam-dong, Seo-gu, Busan 602-702, Korea. Email: mipark@ns.kosinmed.or.kr

doi:10.4021/gr351w gastric adenoma based on IMVPs classifications and the presence of corkscrews.

Keywords: Adenoma; Gastric cancer; Narrow band imaging

\section{Introduction}

It is imperative for an endoscopist to make a differential diagnosis between benign and malignant lesions. For such purposes, magnification endoscopy is a powerful tool for correctly diagnosing superficial neoplasm throughout the entire gastrointestinal tract [1-3]. In addition to the minute structure of the mucosa, the microvascular pattern in the cancerous lesion itself is very important for determining a tumor's malignant potential [4]. The narrow band imaging (NBI) system is an innovative optical technology that can provide clear imaging of the microvascular structure in the mucosal layer. It illuminates the tissue surface through special filters that narrow the red, green, and blue bands with increased intensity of the blue band to enhance the tissue microvascular structure as a result of the differential optical absorption of light by hemoglobin [5]. NBI, in combination with a magnifying endoscope, (MENBI) can yield very clear images of microvessels on mucosal surfaces [6]. According to the Paris classification, superficial gastrointestinal neoplasms can divided into 3 types; superficial elevated (0-IIa) type, superficial flat (0-IIb) type, and superficial depressed (0-IIc) type [7]. Among these three types, it is not always possible for an endoscopist to differentiate early gastric cancer from adenoma in 0-IIa type neoplasms. Because tissue biopsy can only evaluate a tiny piece of the superficial layer of the tumor, it is unable to determine the entire nature of a tumor [8]. Application of endoscopic submucosal dissection (ESD) or endoscopic mucosal resection has made it possible to definitively diagnose many preoperative biopsy-demonstrated adenomas as carcinomas [9, 10]. Changes of diagnosis from adenoma to carcinoma, after ESD, require further investigation and a change in therapeutic strategies. Moreover, in practice, many cases that are diagnosed as adenoma are merely monitored [11]. Therefore, the ability to differentiate between carcino- 

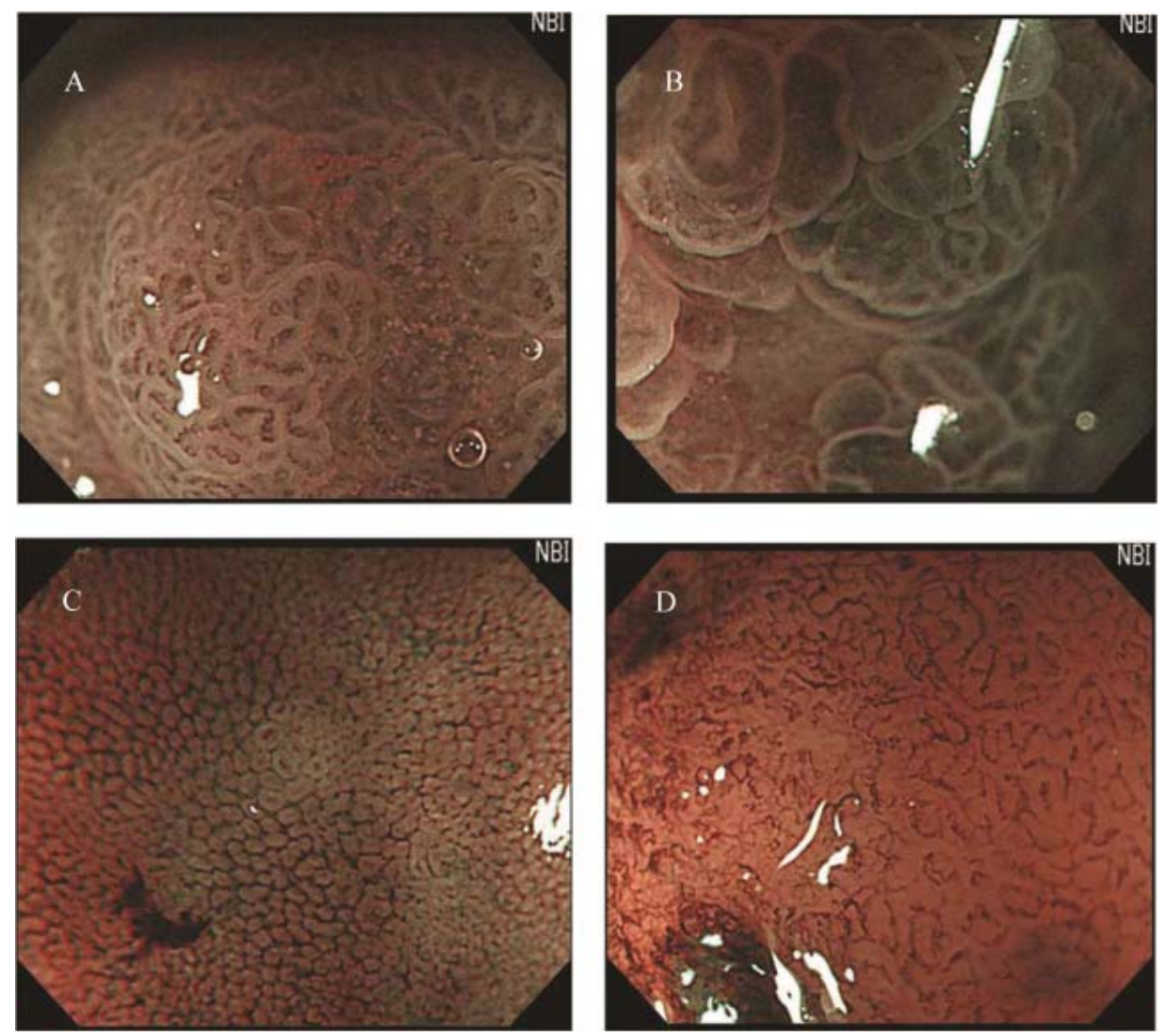

Figure 1. Classification of irregular microvascular patterns observed by MENBI: (A) Slight ISIMVPs, defined by the presence of tiny spiral blood vessels within the fine lobular superficial structure; (B) Severe ISIMVPs, defined by the presence of vertical spiral blood vessels within the coarse lobular superficial structure; (C) Fine networks, defined by fine tubular structures surrounded by a thin microvasculature; (D) Corkscrew patterns, defined by obliterated surface structures and irregular vascular patterns without loop formation. (MENBI: narrow band imaging system in combination with a magnifying endoscope; ISIMVPs: intrastructual irregular microvascular patterns)

ma and adenoma is crucial in establishing appropriate therapeutic strategies for adenomatous lesions. For providing one answer for this problem, we aimed to evaluate the efficacy of MENBI for differentiating gastric carcinoma from adenoma in superficial elevated (0-IIa) lesions by acquiring detailed MENBI examinations.

\section{Materials and Methods}

\section{Patients and specimens}

This study involved retrospective evaluation using our ESD database. Forty-six neoplasms were obtained from patients who had undergone ESD for 0-IIa type lesions according to the Paris endoscopic classification at the Gospel Hospital, Kosin University College of Medicine, Busan, Korea during the period from May 2009 to December 2010. Of these 46 lesions, 32 and 14 lesions, respectively, were postoperatively confirmed to be adenoma and carcinoma. Lesions diagnosed as mucosal low-grade neoplasia of category 3 according to

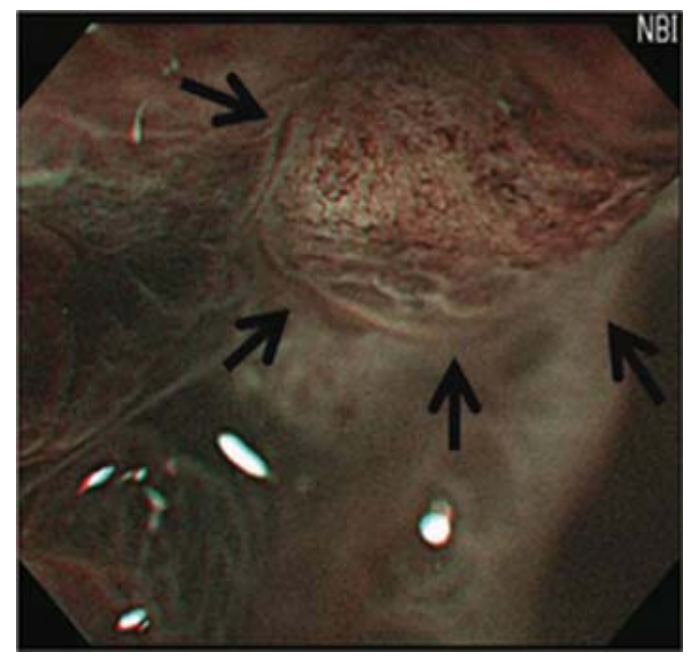

Figure 2. Image of the presence of corkscrews. (The presence of corkscrews occupying over $10 \%$ of the observed field is demonstrated (black arrows). Corkscrew was defined by obliterated surface structures and irregular vascular patterns without loop formation) 
Table 1. Gross Appearance of 0-lla Lesions

\begin{tabular}{llll}
\hline & \multicolumn{1}{c}{ Adenoma $(\mathbf{n}=\mathbf{3 2})$} & EGC IIa $(\mathbf{n}=\mathbf{1 4})$ & P value \\
\hline Size, mean \pm SD, cm & $1.96 \pm 0.80$ & $2.02 \pm 0.53$ & 0.892 \\
Reddish color, n (\%) & $3(9)$ & $2(14)$ & 0.633 \\
Obscure margin, n (\%) & $1(3)$ & $1(7)$ & 0.521 \\
Central concavity, n (\%) & $4(13)$ & $12(86)$ & $<0.001$ \\
\hline
\end{tabular}

EGC Ila: type Ila early gastric cancer.

All analyses were performed by the 2-sided Fisher's exact test except tumor size (Student t-test).

*Sensitivity $85.7 \%$, specificity $87.5 \%$, and positive predictive value $75.0 \%$ (for EGC IIa).

the revised Vienna classification were defined as gastric adenomas [12]. If a whole part of the resected lesion was pathologically diagnosed as intramucosal carcinoma of category 4.2 according to the revised Vienna classification, the lesion was defined as a gastric carcinoma. Each of the patients gave his or her written informed consent. The Ethical Committee of the Kosin University College of Medicine approved the study.

\section{Postoperative examination with MENBI}

Preoperative endoscopic examinations were performed by conventional endoscopy and chromoendoscopy. The endoscopic examinations and ESD were performed by a single expert endoscopist. Endoscopy was performed using an upper-GI endoscope (H260Z, Olympus, Tokyo, Japan). The gross findings, such as lesion color, margin, size, and central concavity, of 0-IIa lesions were evaluated by nonzoom endoscopic observation. ESD was performed for all lesions using an insulation tipped diathermic knife (KD-610L, Olympus, Tokyo, Japan) to remove tumors with a minimum lateral margin of $5 \mathrm{~mm}$. Just after removal of the adenomatous lesions, we performed MENBI (GIF-H260Z; Olympus, Tokyo, Japan; CLV 260SL, Olympus, Tokyo, Japan) on fresh specimens. We screened the complete lesion and picked the three sites, presumably highest grade alteration for MENBI. IMVP cases were subcategorized as (1) slight intrastructual irregular microvascular patterns (ISIMVPs), defined by the presence of thin spiral blood vessels within the fine lobular superficial structure, (2) severe ISIMVPs, defined by the presence of vertical spiral blood vessels within the coarse lobular superficial structure, (3) fine networks, defined by fine tubular structures surrounded by thin microvasculature, or (4) corkscrew patterns, which appear as obliterated surface structures and irregular vascular patterns without loop formation (Fig. 1). Adding to these classifications, we evaluated the presence of corkscrew vascular patterns. The presence of corkscrews was defined by the typical presence of corkscrew vascular patterns in at least over $10 \%$ of the observed field (Fig. 2). The resected tumors were measured and fixed with $10 \%$ buffered formalin. The removed specimens were sectioned at $2 \mathrm{~mm}$ intervals, embedded in paraffin blocks, cut

Table 2. Irregular Microvascular Patterns in 0-Ila Lesions

\begin{tabular}{llll}
\hline IMVPs & Adenoma $(\mathbf{n}=\mathbf{9 6})$ & EGC IIa $(\mathbf{n}=\mathbf{4 2})$ & P value \\
\hline Slight ISIMVPs*, n (\%) & $81(84)$ & $10(24)$ & $<0.001$ \\
Severe ISIMVPs ${ }^{\dagger}$ n (\%) & $6(6)$ & $13(31)$ & $<0.001$ \\
Fine network ${ }^{\ddagger}$, n (\%) & $9(10)$ & $19(45)$ & $<0.001$ \\
Corkscrews, n (\%) & $0(0)$ & $0(0)$ & N.A \\
\hline
\end{tabular}

IMVPS: irregular microvascular patterns; ISIMVPs: intrastructual irregular microvascular patterns; EGC Ila: type Ila early gastric cancer; N.A: not available.

All analyses were performed by the 2-sided Fisher's exact test.

*Sensitivity $84.3 \%$, specificity $76.2 \%$, and positive predictive value $89.0 \%$ (for adenoma).

†Sensitivity $30.9 \%$, specificity $93.5 \%$, and positive predictive value $68.4 \%$ (for EGC Ila).

¥Sensitivity $45.2 \%$, specificity $90.6 \%$, and positive predictive value $67.8 \%$ (for EGC IIa). 
Table 3. The Presence of Corkscrews in 0-Ila Lesions

\begin{tabular}{llll}
\hline The presence of corkscrews & Adenoma $(\mathbf{n}=\mathbf{9 6})$ & EGC IIa $(\mathbf{n}=\mathbf{4 2})$ & P value \\
\hline Positive, n (\%)* & $0(0)$ & $4(9.5)$ & 0.008 \\
Negative, $\mathrm{n}(\%)$ & $96(100)$ & $38(88)$ & \\
\hline
\end{tabular}

EGC Ila: type Ila early gastric cancer.

The definition of the presence of corkscrew is the observation of corkscrew vascular changes in over $25 \%$ of a selected field. All analyses were performed by the 2-sided Fisher's exact test.

*Sensitivity 9.5\%, specificity 100\%, and positive predictive value 100\% (for EGC Ila).

into $5 \mathrm{~mm}$ sections, and stained with hematoxylin and eosin for pathologic diagnosis.

\section{Statistical analysis}

Statistical analysis was performed using the Statistical Package for the Social Sciences (SPSS) software (version 16.0, SPSS, Chicago, IL, USA). The relationship between IMVP and pathologic diagnosis was evaluated using the 2-sided Fisher exact test. The differences between GA and EGC IIa were assessed by 2-sided Fisher exact test and Student t-test. Diagnostic efficacy was assessed by specificity and sensitivity. Two-tailed $\mathrm{P}$ values less than 0.05 were regarded as statistically significant.

\section{Results}

All lesions were removed by ESD en bloc with a full margin distance to enable subsequent accurate pathologic diagnosis. The average tumor diameter was $1.96 \mathrm{~cm}$ in the adenoma group $(\mathrm{n}=32$ ) and $2.02 \mathrm{~cm}$ in the carcinoma group ( $\mathrm{n}=$ 14). The majority of lesions in both groups were discolored, although reddish lesions were seen in $9 \%$ of cases in the adenoma group and in $14 \%$ in the carcinoma group. A central concavity was found in $13 \%$ of the adenoma group cases and in $86 \%$ of the carcinoma cases $(\mathrm{P}<0.001$; Table 1$)$. There were no differences in tumor size, lesion color, or the characteristics of margin between the adenoma group and the carcinoma group: $\mathrm{P}=0.082, \mathrm{P}=0.633$, and $\mathrm{P}=0.521$ respectively; Table 1).

Regarding IMVP subcategories observed by MENBI, by proportions of lesions, (1) slight ISIMVPs accounted for $84 \%$ (81 out of 96), (b) severe ISIMVPs accounted for 6\% (6 out of 96), (3) fine networks accounted for $10 \%$ (9 out of 96), and (4) corkscrews accounted for 0 (0 out of 96) of cases in the adenoma group. The corresponding proportions in the carcinoma group were (1) $24 \%$ (10 out of 42 ), (2) $31 \%$ (13 out of 42), (3) 45\% (19 out of 42), and (4) 0 (0 out of 42). Slight ISIMVPs were significant findings for the adenoma group $(\mathrm{P}<0.001$, sensitivity $84.3 \%$, specificity $76.2 \%$, and positive predictive value $89.0 \%$ ), and severe ISIMVPs and fine networks were significant findings for the EGC IIa group ( $\mathrm{P}<0.001$, sensitivity $30.9 \%$, specificity $93.5 \%$, and positive predictive value $68.4 \%$ for severe ISIMVPs; $\mathrm{P}$ $<0.001$, sensitivity $45.2 \%$, specificity $90.6 \%$, and positive predictive value $67.8 \%$ for severe fine networks; Table 2). The presence of corkscrews was observed more frequently in the EGC IIa group $(\mathrm{P}=0.008$, sensitivity $9.5 \%$, specificity $100 \%$, and positive predictive value $100 \%$; Table 3 ), and was

Table 4. The Presence of Corkscrews and IMVPs

\begin{tabular}{llll}
\hline \multirow{2}{*}{ IMVPs } & \multicolumn{2}{l}{ The presence of corkscrews } & P value \\
\cline { 2 - 4 } & Positive & Negative & \\
\hline Slight ISIMVPs, $\mathrm{n}=91(\%)$ & $0(0)$ & $91(100)$ & 0.012 \\
Severe ISIMVPs, $\mathrm{n}=19(\%)$ & $4(21)$ & $15(79)$ & $<0.001$ \\
Fine network, $\mathrm{n}=28(\%)$ & $0(0)$ & $28(0)$ & 0.582 \\
\hline
\end{tabular}

IMVPS: irregular microvascular patterns; ISIMVPs: intrastructual irregular microvascular patterns; EGC Ila: type Ila early gastric cancer.

All analyses were performed by the 2-sided Fisher's exact test. 
correlated with severe ISIMVPs (Table 4).

\section{Discussion}

The normal patterns of the surface pits and microcirculation are altered in cancerous mucosa [13, 14]. Magnifying endoscopic images, with regard to the minute structure, corresponded well with the histopathological results and emphasized the importance of the tumor's microvascular pattern in addition to the minute structure [15]. The NBI system is a specialized tool for visualizing mucosal microvascular patterns with a narrow band wave length. One of the greatest advantages of MENBI is that it enhances visualization of the mucosal structures and the microvascular patterns thereby providing a more detailed assessment of characteristics of a neoplasm than any other practical modalities. Indeed, in the present study, MENBI-enhanced magnified images of the lesions enable clear visualization of IMVPs. This observation is consistent with the results of other MENBI studies of early stage gastric cancer $[6,16]$.

According to 3 previous studies on the differential diagnosis of gastric carcinoma from adenomatous lesions using MENBI, the investigation of both superficial structure and vascular patterns is useful for the differential diagnosis of adenoma and carcinoma $[6,16,17]$. Nakamura et al analyzed 14 cases and randomly selected 5 points from each case to compensate for the relatively small number of samples [16]. This study showed that slight ISIMVPs were important findings specific to adenoma, and severe ISIMVPs or fine network patterns were highly specific to EGC IIa [16]. These findings fairly coincide with our results (Table 2). While Nakamura et al used special method, 5 sites per lesion for MENBI, to compensate the limitations of small numbers of cases, the small sample size cannot but predicate the lack of statistical significance. Moreover, their method neglected the advantage of endoscopy over histology: the ability to be able to screen the complete lesion and pick the presumably highest grade alteration for evaluation. Our larger scale investigation based on 46 cases with random selection of 3 points, presumably highest grade alteration, from each 0-IIa lesion strongly supports the finding of the previous study that MENBI can be used to differentiate EGC IIa from adenoma based on IMVPs.

Apart from the classical categories of IMVPs defined by Nakamura et al, we also assessed the presence of corkscrews and revealed that the presence of corkscrews can be also helpful to draw a sharp line between EGC IIa and adenoma $(\mathrm{P}=$ 0.008 ; Table 3). Moreover, the presence of corkscrews was only observed with severe ISIMVPs in the EGC IIa group. Corkscrews were not observed in 0-IIa lesions, but were often found in 0-IIc or 0-IIb lesions. This may suggest that severe ISIMVPs are related to more advanced vascular changes than fine networks in relation with carcinoma (Table 4).
In gross findings, it was highly suggested that central concavities may help distinguish between adenoma and EGC IIa (Table 1). However, reddish lesions and obscure margin, thought to indicate EGC IIa [18] were neither specific for EGC IIa nor adenoma.

The use of ESD for treating adenoma as well as early gastric cancer has currently been popularized with rapid technical progress. Postoperative pathologic evaluation revealed that a few 0 -IIa lesions, diagnosed as adenoma by preoperative forcep biopsies, contain carcinomatous components. Adenomatous lesions have often been observed due to the idea that the malignant potential is relatively low from a long-term report [19]. However, just following up on 0-IIa adenomatous lesions that have carcinomatous foci can make it possible to miss the right time for ESD. This is the reason why accurate diagnosis is crucial for 0-IIa lesions. In this light, our result can be applied not only to acquire an accurate impression by observing IMVPs, but also to perform precise target biopsies from potential cancerous portions on 0-IIa lesions.

In conclusion, MENBI can be used as a highly effective tool to differentiate EGC IIa from gastric adenoma based on IMVP classification and the presence of corkscrews in 0 -IIa lesions that are difficult to distinguish by conventional endoscopy. Moreover, it can also be used for precise target biopsies to avoid missing cancerous components. Severe ISIMVPs may reflect more advanced vascular changes than fine networks and slight ISIMVPs. This theory needs to be verified by pathologic evaluation with MENBI examination in the future.

\section{Acknowlegement}

This study was supported by a grant from Kosin University College of Medicine (2009).

\section{Conflict of Interests}

The authors report no conflicts of interest. The authors alone are responsible for the content and writing of the paper.

\section{References}

1. Kara MA, Ennahachi M, Fockens P, ten Kate FJ, Bergman JJ. Detection and classification of the mucosal and vascular patterns (mucosal morphology) in Barrett's esophagus by using narrow band imaging. Gastrointest Endosc. 2006;64(2):155-166.

2. Kudo S, Tamura S, Nakajima T, Yamano H, Kusaka $\mathrm{H}$, Watanabe H. Diagnosis of colorectal tumorous lesions by magnifying endoscopy. Gastrointest Endosc. 
1996;44(1):8-14.

3. Yao K, Oishi T, Matsui T, Yao T, Iwashita A. Novel magnified endoscopic findings of microvascular architecture in intramucosal gastric cancer. Gastrointest Endosc. 2002;56(2):279-284.

4. Tajiri H, Matsuda K, Fujisai J. What can we see with the endoscope? Present status and future perspectives. Dig Endosc 2002;14:131-137.

5. Kuznetsov K, Lambert R, Rey JF. Narrow-band imaging: potential and limitations. Endoscopy. 2006;38(1):76-81.

6. Nakayoshi T, Tajiri H, Matsuda K, Kaise M, Ikegami M, Sasaki H. Magnifying endoscopy combined with narrow band imaging system for early gastric cancer: correlation of vascular pattern with histopathology (including video). Endoscopy. 2004;36(12):1080-1084.

7. The Paris endoscopic classification of superficial neoplastic lesions: esophagus, stomach, and colon: November 30 to December 1, 2002. Gastrointest Endosc. 2003;58(6 Suppl):S3-43.

8. Fertitta AM, Comin U, Terruzzi V, Minoli G, Zambelli A, Cannatelli G, Bodini P, et al. Clinical significance of gastric dysplasia: a multicenter follow-up study. Gastrointestinal Endoscopic Pathology Study Group. Endoscopy. 1993;25(4):265-268.

9. Yamamoto H. Endoscopic submucosal dissection of early cancers and large flat adenomas. Clin Gastroenterol Hepatol. 2005;3(7 Suppl 1):S74-76.

10. Tamai N, Kaise M, Nakayoshi T, Katoh M, Sumiyama K, Gohda K, Yamasaki T, et al. Clinical and endoscopic characterization of depressed gastric adenoma. Endoscopy. 2006;38(4):391-394.

11. Nakamura M, Shibata T, Tahara T, Yoshioka D, Okubo M, Mizoguchi Y, Kuroda M, et al. The usefulness of magnifying endoscopy with narrow-band imaging to distinguish carcinoma in flat elevated lesions in the stomach diagnosed as adenoma by using biopsy samples. Gastrointest Endosc. 2010;71(6):1070-1075.

12. Dixon MF. Gastrointestinal epithelial neoplasia: Vienna revisited. Gut. 2002;51(1):130-131.

13. Honmyo U, Misumi A, Murakami A, Mizumoto S, Yoshinaka I, Maeda M, Yamamoto S, et al. Mechanisms producing color change in flat early gastric cancers. Endoscopy. 1997;29(5):366-371.

14. Caruso RA, Speciale G, Inferrera A, Rigoli L, Inferrera C. Ultrastructural observations on the microvasculature in advanced gastric carcinomas. Histol Histopathol. 2001;16(3):785-792.

15. Tajiri H, Doi T, Endo H, Nishina T, Terao T, Hyodo I, Matsuda K, et al. Routine endoscopy using a magnifying endoscope for gastric cancer diagnosis. Endoscopy. 2002;34(10):772-777.

16. Yao K, Iwashita A, Tanabe H, Nishimata N, Nagahama T, Maki S, Takaki Y, et al. White opaque substance within superficial elevated gastric neoplasia as visualized by magnification endoscopy with narrow-band imaging: a new optical sign for differentiating between adenoma and carcinoma. Gastrointest Endosc. 2008;68(3):574580.

17. Jung MK, Jeon SW, Park SY, Cho CM, Tak WY, Kweon YO, Kim SK, et al. Endoscopic characteristics of gastric adenomas suggesting carcinomatous transformation. Surg Endosc. 2008;22(12):2705-2711.

18. Kamiya T, Morishita T, Asakura H, Miura S, Munakata Y, Tsuchiya M. Long-term follow-up study on gastric adenoma and its relation to gastric protruded carcinoma. Cancer. 1982;50(11):2496-2503. 